\title{
Tau Reconstruction, Identification Algorithms and Performance in ATLAS
}

\author{
Margar Simonyan \\ On behalf of the ATLAS Collaboration. \\ Niels Bohr Institute, Copenhagen University. \\ Blegdamsvej 17, 2100 Copenhagen, Denmark
}

\begin{abstract}
Tau leptons play an important role in the physics program at the LHC. They are used not only in searches for new phenomena like the Higgs boson or Supersymmetry and electroweak measurements but also in detector related studies like the determination of the missing transverse energy scale. Optimal identification of hadronically decaying tau leptons is achieved by using detailed information from tracking and calorimeter detector components. Variables describing the properties of calorimeter energy deposits and track reconstruction within tau candidates are combined in multi-variate discriminants, to achieve high rejection against backgrounds, which is challenging in a high particle density environments. The identification efficiencies are measured in $W \rightarrow \tau \nu$ and $Z \rightarrow \tau \tau$ events, and compared with the prediction of the Monte Carlo simulation. The energy scale uncertainties for tau leptons are determined by investigating single hadron calorimeter response, as well as kinematic distributions in $Z \rightarrow \tau \tau$ events.
\end{abstract}

Keywords: ATLAS, tau lepton

\section{Introduction}

Tau leptons are important signatures for Standard Model processes and searches for new physics. With a mass of $1.777 \mathrm{GeV}[1]$, the tau is the heaviest lepton and due to its short lifetime of $2.9 \times 10^{-13} \mathrm{~s}$ $(c \tau=87 \mu \mathrm{m})$ decays inside the beam pipe of the LHC. The tau lepton is the only lepton that has a hadronic decay mode. The branching ratio for leptonic decays is approximately 0.35 and hadronic decay modes account for the remaining $65 \%$. The majority of hadronic tau decays are characterized by one or three charged pions possibly accompanied by neutral pions. The cross-section of most of the Standard Model and new physics processes with tau leptons in the final state are small compared to the overwhelming background from multijet production at LHC. Therefore well performing tau identification is crucial.

The ATLAS detector is a large collider detector built to be used for LHC collisions at CERN. It consists of inner detector combining different tracking

Email address: Margar.Simonyan@cern.ch (Margar Simonyan)

Preprint submitted to Nuc. Phys. (Proc. Suppl.) subsystems, sampling calorimeters and muon spectrometer.

In ATLAS [2] tau reconstruction and identification concentrates on the hadronic decay modes of a tau lepton. The number of tracks within $\Delta R=\sqrt{(\Delta \eta)^{2}+(\Delta \phi)^{2}}<0.2^{1}$ is used to classify the tau candidate into 1 or multi-prong categories. These decays can be differentiated from QCD jets by their characteristics, such as low track multiplicity, collimated energy deposits, and in the case of 3-prong tau decays the displacement of the secondary vertex.

\section{Reconstruction}

The reconstruction of hadronically decaying tau leptons is seeded by calorimeter jets reconstructed

\footnotetext{
${ }^{1}$ The ATLAS experiment uses a right-handed coordinate system with its origin at the nominal interaction point (IP) in the center of the detector and the $\mathrm{z}$-axis along the beam pipe. The $\mathrm{x}$-axis points from the IP to the center of the LHC ring, and the $\mathrm{y}$-axis points upward. The $\phi$ and $\theta$ angles are the azimuthal and polar angles. The pseudorapidity is defined by $\eta=-\ln \tan \theta / 2$.
} 


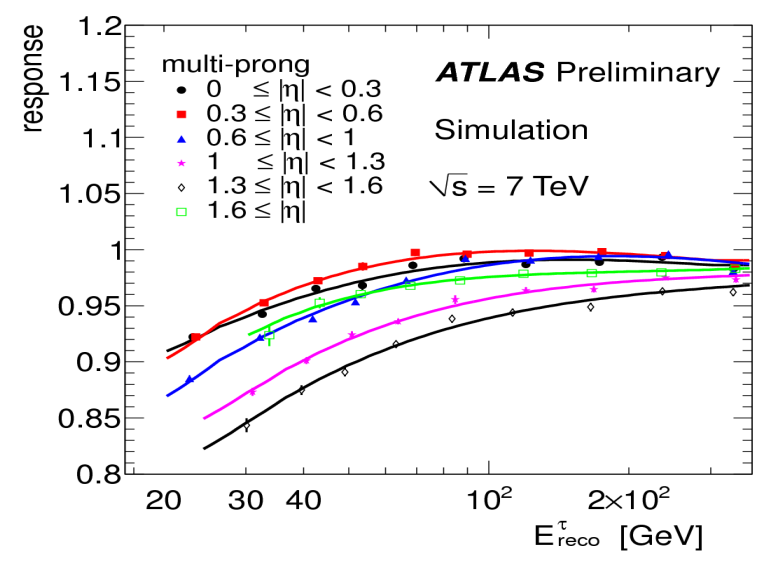

Figure 1: Calibration functions [6] for multi-prong tau candidates.

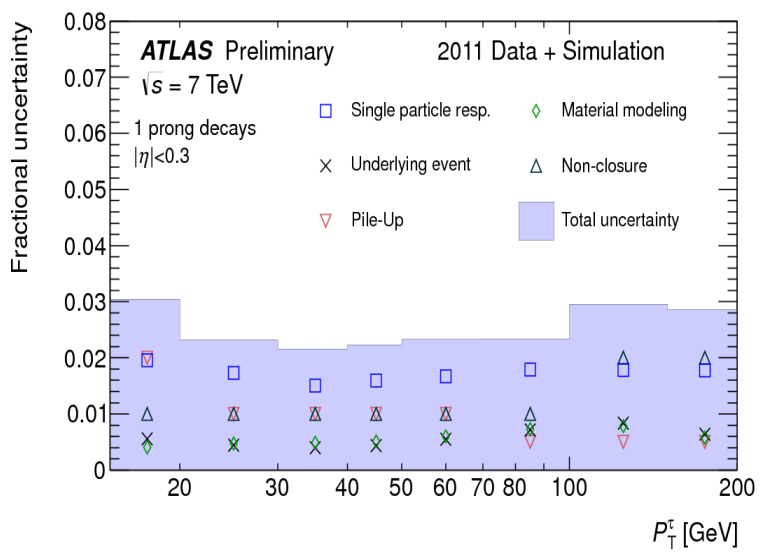

Figure 2: The energy scale uncertainties [6] for 1-prong tau candidates.

with the anti $-k_{t}$ algorithm [3] using a radius parameter $R=0.4$. Topological clusters [4] made of calorimeter cells calibrated using the Local Hadron Calibration (LC) [5] are used as an input for the jet algorithm. The tau reconstruction is run on all seed jets with $p_{\mathrm{T}}>10 \mathrm{GeV}$ and within $|\eta|<2.5$, the $\eta$-range of the ATLAS tracking system. The tau energy is calculated using all calorimeter clusters within a core of $\Delta R=0.2$ around the vector sum of clusters associated with the jet seed. Calibration factors are derived $[6,7]$ from response functions using Monte Carlo simulations. Response functions depend on the tau energy reconstructed at LC scale, and calculated separately for 1 and multi-prong tau leptons, as well as for different detector regions. The response functions for multiprong taus are shown in Fig. 1. The systematic un-

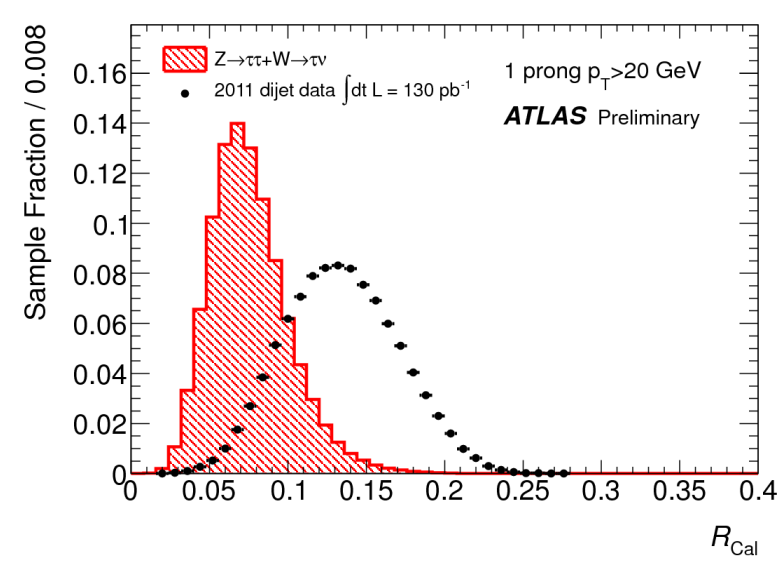

Figure 3: The distribution of $R_{C a l}$ observable [8] for 1prong tau candidates.

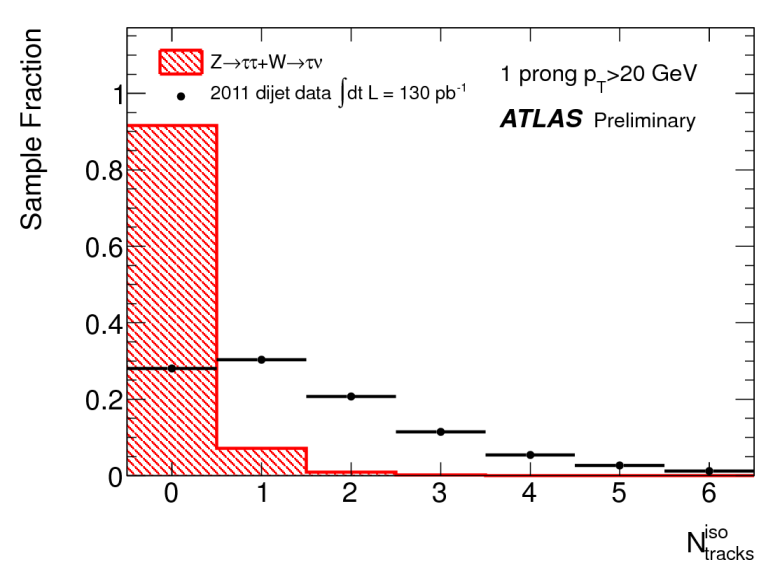

Figure 4: The distribution of track multiplicity [8] in ring $0.2<\Delta R<0.4$ for 1 -prong tau candidates.

certainties on the tau energy scale are derived from in situ measurement as well as Monte Carlo simulations. The uncertainty on the tau energy scale for 1-prong candidates is shown in Fig 2, the scale is known with $3 \%$ precision in the entire $p_{\mathrm{T}}$ range.

\section{Identification}

Since there is no attempt to separate QCD jets and tau leptons in the reconstruction process a dedicated identification step is needed. It is based on variables which provide discrimination power between QCD jets and tau leptons. Typical detector signatures for the tau leptons are collimated energy deposits in the calorimeter and one or three tracks. Several observables employing these characteristics 


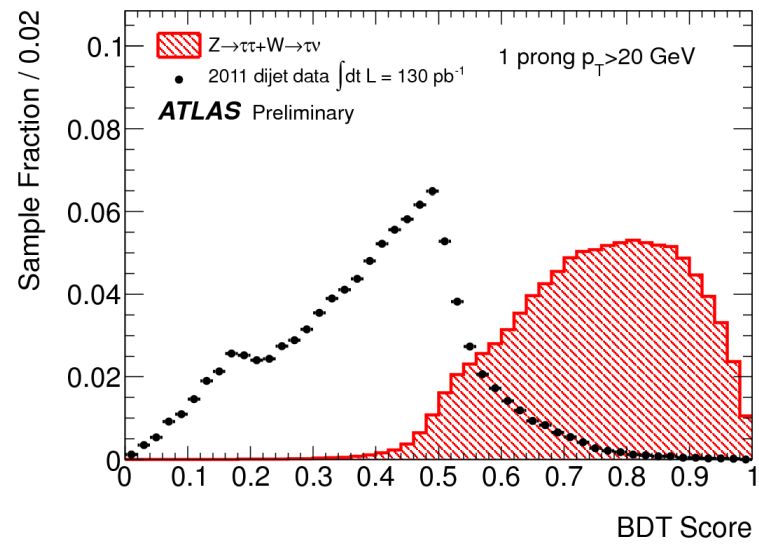

Figure 5: BDT score distribution [8] for 1-prong tau candidates.

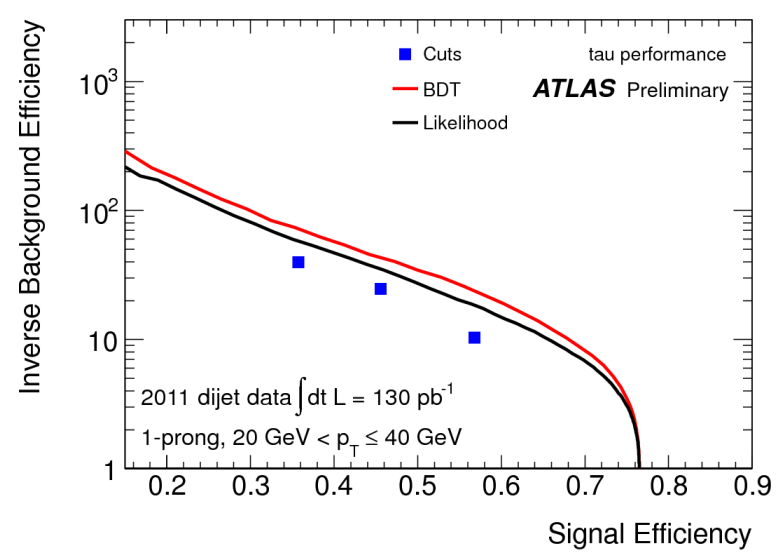

Figure 6: The inverse background efficiency as a function of signal efficiency [8] for 1-prong taus.

are calculated using the calorimeter and tracking information. A few examples are presented below. An observable describing the width of energy deposits in the calorimeter $\left(R_{c a l}\right)$ defined as

$$
R_{C a l}=\frac{\sum_{i \in\{\text { all }\}}^{\Delta R_{i}<0.4} E_{\mathrm{T}, i} \Delta R_{i}}{\sum_{i \in\{\text { all }\}}^{\Delta R_{i}<0.4} E_{\mathrm{T}, i}},
$$

where $E_{\mathrm{T}, i}$ are the transverse energy of cells in $\Delta R<0.4$, is used to separate taus and jets. The distribution of $R_{C a l}$ for 1-prong taus and QCD jets is shown in Fig. 3.

The track multiplicity distribution in the ring $0.2<\Delta R<0.4$ has a separation power as can be seen in Fig. 4 and is used for tau identification. Several of such observables are combined in

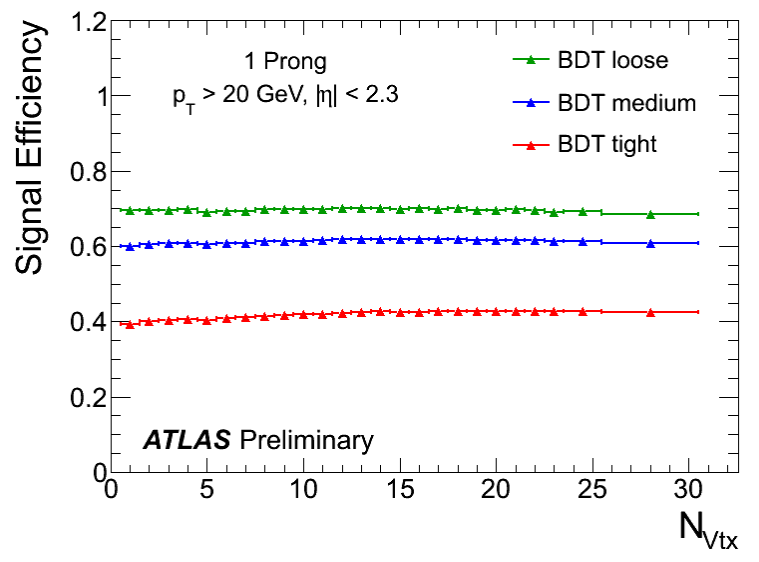

Figure 7: Tau identification efficiency as a function of primary vertex candidate multiplicity [9].

multi-variate techniques to obtain the best possible separation between tau leptons and QCD jets. A projective likelihood (LLH) using the likelihoodratio of signal and background, and boosted decision trees (BDT) are currently in use. The methods use different sets of identification variables and are separately trained for 1 and multi-prong tau candidates. The BDT output score is shown in Fig. 5 for 1-prong tau candidates. For the training of the identification algorithms, the jet background was obtained from data, while the tau decay signal was simulated in $W \rightarrow \tau \nu$ and $Z \rightarrow \tau \tau$ Monte Carlo samples. The inverse background efficiency as a function of signal efficiency for the two methods is shown in Fig. 6 for 1-prong tau candidates.

Three working points (loose, medium, tight) corresponding to different signal efficiencies and background rejection are provided for the tau identification methods. The signal efficiency for the working points of BDT identification is shown to be independent of the number of reconstructed primary vertex candidates in Fig. 7.

Electrons can also be misidentified as a tau lepton. Due to the signature of the electron in the detector, they will be reconstructed mostly as a 1prong tau candidate. To distinguish between electrons and tau leptons discriminating observables are combined into a boosted decision tree and good performance is achieved.

\section{Identification Efficiency Measurements}

The performance and systematic uncertainties of the tau identification methods are evaluated on 


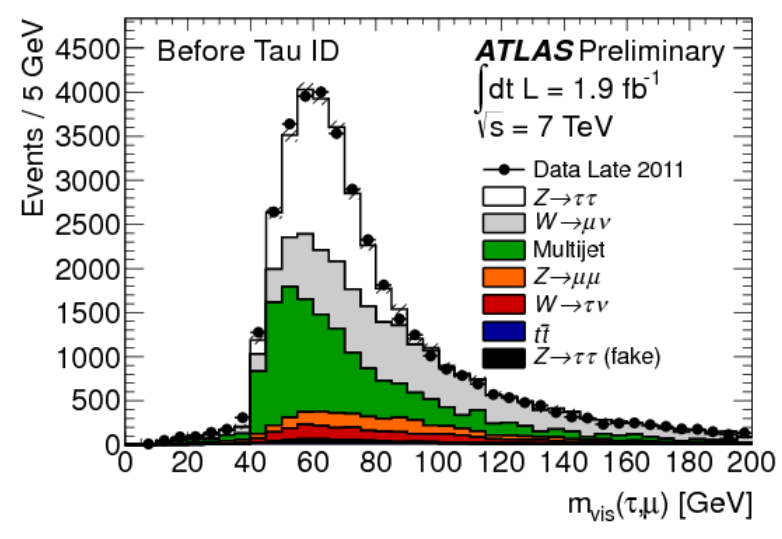

Figure 8: Muon-tau pair mass distribution [8, 9] before tau identification.

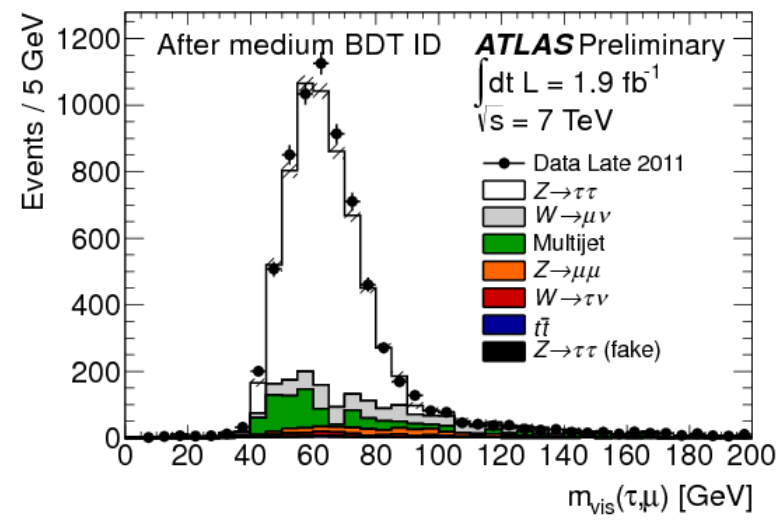

Figure 9: Muon-tau pair mass distribution $[8,9]$ after tau identification.

data using two different signal channels. The first method uses $Z \rightarrow \tau \tau$ events and relies on a tagand-probe [8] approach. Events are tagged with a muon from a tau decay, and the other tau lepton in the event is required to decay hadronically, forming the probe that is used to measure the identification efficiency. The background is dominated by $W \rightarrow \tau \nu$ and was estimated from Monte Carlo simulation, while the multi-jet background was obtained by a data-driven method.

The mass of the muon and the hadronic tau pair is shown in Fig. 8 before and in Fig. 9 after applying the medium BDT tau identification. The tau identification efficiency was also measured using $W \rightarrow \tau \nu$ events [10] collected in $1.9 \mathrm{fb}^{-1}$ of data. Variables based on the missing transverse energy were used to select the events. The number of hadronic tau candidates is derived by fitting templates built with distributions of track multiplicity of the tau candidates. Three different templates were used: real hadronic tau decays, electrons misidentified as tau leptons, and jets misidentified as tau leptons. While the first two are obtained from Monte Carlo simulation, the jet template was estimated from a data control region. The measured efficiencies in both methods are in good agreement with Monte Carlo predictions.

\section{Conclusions}

ATLAS tau reconstruction is well understood. An energy calibration independent of the number of overlapping interactions has been developed achieving $3 \%$ uncertainties on the energy scale. Identification algorithms provide high rejection against jets and electrons already at medium signal efficiencies. The performance of the identification algorithms is measured in data using in situ techniques and good agreement between data and $\mathrm{MC}$ is obtained.

\section{References}

[1] J. Beringer et al. (Particle Data Group), Phys. Rev. D86, 010001 (2012).

[2] The ATLAS Collaboration, The ATLAS Experiment at the CERN Large Hadron Collider, JINST 3 (2008) S08003.

[3] M. Cacciari, et al., The anti- $k_{t}$ jet clustering algorithm, Journal of High Energy Physics 2008 (04) (2008) 063.

[4] W. Lampl, et al., Calorimeter Clustering Algorithms: Description and Performance. ATL-LARG-PUB-2008002.

[5] T. Barillari et al., Local Hadronic Calibration. ATLLARG-PUB-2009-001-2.

6] The ATLAS Collaboration, Determination of the tau energy scale and the associated systematic uncertainty in proton-proton collisions at $\sqrt{s}=7 \mathrm{TeV}$ with the ATLAS detector at the LHC in 2011, Tech. Rep. ATLASCONF-2012-054, CERN, Geneva (Jun 2012).

[7] The ATLAS Collaboration, Reconstruction, energy calibration, and identification of hadronically decaying tau leptons, Tech. Rep. ATLAS-CONF-2011-077, CERN, Geneva (May 2011).

[8] The ATLAS Collaboration, Performance of the Reconstruction and Identification of Hadronic Tau Decays with ATLAS, Tech. Rep. ATLAS-CONF-2011-152, CERN, Geneva (Nov 2011).

[9] The ATLAS Collaboration, ATLAS Tau Public Results.

[10] The ATLAS Collaboration, Measurement of hadronic tau decay identification efficiency using $W \rightarrow \tau \nu$ events, Tech. Rep. ATLAS-CONF-2011-093, CERN, Geneva (Jul 2011). 